\title{
Thermal Convective Instability in an Oldroyd-B Nanofluid Saturated Porous Layer
}

\author{
I.S. Shivakumara ${ }^{\mathrm{a}}, \mathrm{M}$. Dhananjaya ${ }^{\mathrm{b}}$, Chiu-On $\mathrm{Ng}^{\mathrm{c} *}$ \\ ${ }^{a}$ Department of Mathematics, Bangalore University, Bangalore 560 001, India \\ ${ }^{\mathrm{b}}$ Department of Mathematics, Bangalore Institute of Technology, Bangalore 560 004, India \\ ${ }^{c}$ Department of Mechanical Engineering, The University of Hong Kong, Pokfulam Road, \\ Hong Kong.
}

\begin{abstract}
The onset of convective instability in a layer of porous medium saturated by the Oldroyd-B viscoelastic nanofluid heated from below is investigated by incorporating the effects of Brownian diffusion and thermophoresis. The flux of volume fraction of nanoparticles is taken to be zero on the boundaries. The resulting eigenvalue problem is solved numerically using the Galerkin method. The onset of convective instability is oscillatory only if the strain retardation parameter is less than the stress relaxation parameter and also when the strain retardation parameter does not exceed a threshold value which in turn depends on other physical parameters. The oscillatory onset is delayed with increasing strain retardation parameter, while an opposite trend is noticed with increasing stress relaxation parameter. The effect of increasing modified diffusivity ratio, concentration Darcy-Rayleigh number, modified particle density increment and Lewis number is to hasten the onset of stationary and oscillatory convection and also to decrease the ranges of the strain retardation parameter within which oscillatory convection is preferred.
\end{abstract}

Key words: convection, linear stability, porous medium, viscoelastic nanofluid.

*Corresponding author

Email: cong@hku.hk (Chiu-On Ng) 


\section{Nomenclature}

a wave number

$D_{B} \quad$ Brownian diffusion coefficient

$D_{T} \quad$ thermophoretic diffusion coefficient

$d \quad$ depth of the porous layer

$k \quad$ thermal conductivity of the nanofluid

$K \quad$ permeability of the porous medium

Le Lewis number

$l, m \quad$ wave numbers in the $x$ and $y$-directions

$M \quad$ heat capacity ratio

$N_{A} \quad$ modified diffusivity ratio

$N_{B} \quad$ modified particle density increment

$p \quad$ pressure

$\vec{q}=(u, v, w) \quad$ nanofluid velocity

$R_{m} \quad$ basic density Darcy - Rayleigh number

$R_{t} \quad$ thermal Darcy - Rayleigh number

$R_{n} \quad$ nanoparticle concentration Darcy - Rayleigh number

$(x, y, z) \quad$ Cartesian coordinates

$t \quad$ time

$T \quad$ nanofluid temperature

$T_{0} \quad$ temperature at the lower boundary

$T_{1} \quad$ temperature at the upper boundary

W amplitude of perturbed vertical component of velocity

\section{Greek symbols}

$\beta \quad$ the coefficient of thermal expansion

$\varepsilon \quad$ porosity of porous media

$\eta \quad$ thermal expansion coefficient of viscosity

$\kappa \quad$ thermal diffusivity of the fluid

$\lambda_{1} \quad$ constant relaxation time 


$\begin{array}{ll}\lambda_{2} & \text { constant retardation time } \\ \Lambda_{1} & \text { stress relaxation parameter } \\ \Lambda_{2} & \text { strain retardation parameter } \\ \mu & \text { viscosity of the fluid } \\ \omega & \text { growth rate } \\ \phi & \text { nanoparticle volume fraction } \\ \phi_{0} & \text { reference value of nanoparticle volume fraction } \\ \Phi & \text { amplitude of perturbed nanoparticle volume fraction } \\ \rho & \text { nanofluid density } \\ \Theta & \text { amplitude of perturbed temperature }\end{array}$

\section{Superscripts}

$*$

dimensionless variable

perturbed variable

\section{Subscripts}

$\begin{array}{ll}b & \text { basic state } \\ f & \text { fluid } \\ p & \text { particle }\end{array}$

\section{Introduction}

The term 'nanofluid' was first coined by Choi [1] and such a fluid is envisioned to describe a fluid in which nanometer-sized particles (10-100 nm) are stably suspended in conventional heat transfer basic fluids. Materials commonly used for nanoparticles include oxides such as alumina, silica, titania and copper oxide, and metals such as copper and gold. Carbon nanotubes and diamond nanoparticles have also been used to realize nanofluids. Popular base fluids include water, oil and organic fluids such as ethanol, propylene glycol and ethylene glycol. Relative to the base fluid, it has been observed consistently by many researchers that the nanofluids have abnormal thermal conductivity, viscosity and singlephase convective heat transfer coefficient. These fluids are considered to offer important advantages over conventional heat transfer fluids. The recent review articles by Kakac and 
Pramuanjaroenkij [2], Yu and Xie [3], Goharshadi et al. [4], Mahbubul et al. [5] have covered the latest developments in this field in detail.

In recent years, buoyancy driven convection in nanofluids has attracted researchers and has been a subject of intense current interest. Tzou [6, 7] studied buoyancy driven convection in a horizontal nanofluid layer heated from below on the basis of the transport equations developed by Buongiorno [8], while Kim et al. [9] treated the Bénard problem for nanofluids in a different context. Its counterpart in a porous medium, the Darcy- Bénard problem with nanofluids, has also attracted equal importance in the literature because of its importance in many fields of modern science, engineering and technology, chemical and nuclear industries and bio-mechanics. Such an instability problem was first considered by Nield and Kuznetsov [10]. Following this formalism several studies were undertaken subsequently to investigate various additional effects on the problem by the same authors and others. The details can be found in the monograph of Nield and Bejan [11]. In studying these convective instability problems, the volume fraction of nanoparticles was prescribed at the boundaries. Recently, Nield and Kuznetsov [12] pointed out that this type of boundary condition on volume fraction of nanoparticles is physically not realistic as it is difficult to control the nanoparticle volume fraction on the boundaries, and suggested an alternative boundary condition that is, the flux of volume fraction of nanoparticles is zero on the boundaries.

Studies have also revealed that nanofluids containing $\mathrm{SiO}_{2}$ nanoparticles with ethylene glycol and water as base fluids demonstrate a non-Newtonian behavior at low temperatures (Namburu et al. [13]). Besides, Chen et al. [14-16] and Schmidt et al. [17] also indicated the non-Newtonian rheological behavior of nanofluids. Thus, it is imperative to consider non-Newtonian effects in the study of convection in nanofluids. There exist different kinds of non-Newtonian fluids and they do not lend themselves to a unified treatment. Many of the base fluids exhibit viscoelastic behavior and hence considering viscoelastic model is more appropriate than an inelastic type of non-Newtonian model in the study of thermal convective instability in nanofluids. In general, viscoelastic instability is observed in polymer melts as well as in polymer solutions, which usually consist of a Newtonian solvent and a polymeric solute. These solutions are often highly elastic but have an essentially constant viscosity. They are known as Boger fluids and are reasonably well represented by the Oldroyd-B constitutive model (Bird et al. [18], Li and Khayat [19]). The 
Oldroyd-B constitutive model is adopted widely to examine the influence of elasticity on thermal convective instability. This is because the Oldroyd-B model represents adequately highly elastic (Boger) fluids, for which the viscosity remains sensibly constant over a wide range of shear rates. Besides, it is one of the simplest viscoelastic laws that account for normal stress effects which are responsible for the periodic phenomena arising in viscoelastic fluids. More importantly, almost all experimental measurements and flow visualization reported on the instability of viscoelastic flows have been conducted on Boger fluids. Comparison between theory and experiment becomes possible when the Oldroyd-B constitutive equation is used. Of course, there exist more realistic phenomenological or molecular-theory-based models (Bird et al. [18]; Tanner [20]) but they probably lead to a different stability picture (Larson [21]).

Copious literature is available on thermal convection in a layer of porous medium saturated by a viscoelastic regular fluid. Alishaev and Mirzadjanzade [22] were the first to deal with viscoelastic flows in porous media for calculations of delay phenomenon in filtration theory. Rudraiah et al. [23] studied thermal convection in a viscoelastic-fluidsaturated porous layer. A comprehensive review on non-Newtonian fluid flows and heat transfer in porous media is given by Shenoy [24]. Kim et al. [25] investigated thermal instability in a porous layer saturated with viscoelastic fluid and it is found that the overstability is a preferred mode of instability for a certain range of elastic parameters. Malashetty et al. [26] and Shivakumara et al. [27] analyzed the effects of local thermal nonequilibrium on the onset of convection in a viscoelastic-fluid-saturated porous layer. Zhang et al. [28] performed linear and nonlinear thermal stability analyses of a horizontal layer of an Oldroyd-B fluid in a porous medium heated from below. The details can be found in the book by Nield and Bejan [11].

Nonetheless, the study of thermal convective instability in a viscoelastic nanofluid saturated porous layer is comparatively of recent origin and it is still in a rudimentary stage. Sheu [29] studied the onset of convection in a horizontal layer of porous medium saturated with a viscoelastic nanofluid while Yadav et al. [30] extended this study to include the effect of rotation and variations in thermal conductivity and viscosity. In the latter paper a weakly nonlinear stability analysis has also been carried out. To make analytical progress, the volume fraction of nanoparticles is prescribed at the boundaries in the above studies. But it is believed that these conditions are difficult to visualize in practice. Under the circumstances, 
it is desirable to probe the implications of physically realistic boundary conditions as far as the volume fraction of nanoparticles is concerned.

The intent of the present paper is to study the onset of thermal convective instability in an Oldroyd-B type of viscoelastic nanofluid-saturated porous layer considering the flux of volume fraction of nanoparticles is zero at the boundaries as it is physically more realistic (Nield and Kuznetsov [12]). The resulting eigenvalue problem is solved numerically using the Galerkin method and the results are presented graphically.

\section{Mathematical formulation}

The physical configuration is as shown in Fig. 1. We consider a horizontal layer of an incompressible Oldroyd-B nanofluid-saturated Darcy porous medium of thickness $d$. A Cartesian coordinate system $(x, y, z)$ is chosen such that the origin is at the bottom of the porous layer. The gravity is acting in the negative vertical $z$-direction. The lower and upper impermeable boundaries are maintained at constant but different temperatures $T_{0}$ and $T_{1}\left(<T_{0}\right)$, respectively. In investigating the problem, the effects of Brownian motion and thermophoresis are considered.

The governing equations under the Oberbeck-Boussinesq approximation are:

$\nabla \cdot \vec{q}=0$

$\left(1+\lambda_{1} \frac{\partial}{\partial t}\right)(\nabla p-\rho \vec{g})=-\frac{\mu}{K}\left(1+\lambda_{2} \frac{\partial}{\partial t}\right) \vec{q}$

$(\rho c)_{m} \frac{\partial T}{\partial t}+(\rho c)_{f}(\vec{q} \cdot \nabla) T=k \nabla^{2} T+\varepsilon(\rho c)_{p}\left[D_{B} \nabla \phi \cdot \nabla T+\frac{D_{T}}{T_{0}} \nabla T \cdot \nabla T\right]$

$\frac{\partial \phi}{\partial t}+\frac{1}{\varepsilon}(\vec{q} \cdot \nabla) \phi=D_{B} \nabla^{2} \phi+\frac{D_{T}}{T_{0}} \nabla^{2} T$

$\rho=\phi \rho_{p}+(1-\phi) \rho_{f}\left[1-\beta\left(T-T_{0}\right)\right]$

where, $\vec{q}=(u, v, w)$ the velocity vector, $p$ the pressure, $\rho$ the overall density of the nanofluid, $\rho_{p}$ the density of nanoparticles, $\rho_{f}$ the density of base fluid, $\vec{g}$ the acceleration due to gravity, $\mu$ the viscosity of the nanofluid, $K$ the permeability of the porous medium, 
$\varepsilon$ the porosity of the porous medium, $\lambda_{1}$ the relaxation time, $\lambda_{2}$ the retardation time, $T$ the temperature of the nanofluid, $\phi$ the nanoparticle volume fraction, $\beta$ the coefficient of thermal expansion, $c$ the specific heat at constant pressure, $k$ the thermal conductivity, $D_{B}$ the Brownian diffusion coefficient and $D_{T}$ the thermophoretic diffusion coefficient.

We introduce the dimensionless variables as follows:

$$
\begin{aligned}
& \left(x^{*}, y^{*}, z^{*}\right)=\left(\frac{x}{d}, \frac{y}{d}, \frac{z}{d}\right), \vec{q}^{*}=\left(\frac{d}{\kappa}\right) \vec{q}, t^{*}=\left(\frac{\kappa}{\varepsilon d^{2}}\right) t, p^{*}=\left(\frac{K}{\mu \kappa}\right) p, \phi^{*}=\frac{\phi-\phi_{0}}{\phi_{0}} \\
& T^{*}=\frac{T-T_{0}}{T_{0}-T_{1}}
\end{aligned}
$$

where, $\kappa=k /(\rho c)_{f}$. Substituting Eq. (6) into Eqs. (1)-(5) and neglecting the asterisks for simplicity, we obtain

$$
\begin{aligned}
& \nabla \cdot \vec{q}=0 \\
& \left(1+\Lambda_{1} \frac{\partial}{\partial t}\right)\left(\nabla p+R_{m} \hat{k}+R_{n} \phi \hat{k}-R_{t} T \hat{k}\right)=-\left(1+\Lambda_{2} \frac{\partial}{\partial t}\right) \vec{q} \\
& M \frac{\partial T}{\partial t}+(\vec{q} \cdot \nabla) T=\nabla^{2} T+\frac{N_{B}}{L e} \nabla \phi \cdot \nabla T+\frac{N_{A} N_{B}}{L e} \nabla T \cdot \nabla T \\
& \frac{\partial \phi}{\partial t}+(\vec{q} \cdot \nabla) \phi=\frac{N_{A}}{L e} \nabla^{2} T+\frac{1}{L e} \nabla^{2} \phi
\end{aligned}
$$

where, $\Lambda_{1}=\kappa \lambda_{1} / \varepsilon d^{2}$ is the stress relaxation parameter, $R_{m}=d K \beta\left[\phi_{0} \rho_{p}+\left(1-\phi_{0}\right) \rho_{f}\right] g / \mu \kappa$ is the basic density Darcy-Rayleigh number, $R_{t}=d K \beta \rho_{f}\left(T_{0}-T_{1}\right) g / \mu \kappa$ is the thermal Darcy-Rayleigh number, $R_{n}=K d\left(\rho_{p}-\rho_{f}\right) g \phi_{0} / \mu \kappa$ is the concentration Darcy-Rayleigh number, $\Lambda_{2}=\kappa \lambda_{2} / \varepsilon d^{2}$ is the strain retardation parameter, $M=(\rho c)_{m} /(\rho c)_{f} \varepsilon$ is the heat capacity ratio, $N_{A}=D_{T}\left(T_{0}-T_{1}\right) / D_{B} T_{0}$ is the modified diffusivity ratio, $N_{B}=(\rho c)_{p} \phi_{0} /(\rho c)_{f}$ is the modified particle density increment and $L e=\kappa / \varepsilon D_{B}$ is the Lewis number. In obtaining Eq. (8), a term proportional to the product of $\phi$ and $T$ is neglected in the spirit of the Oberbeck-Boussinesq approximation and this is valid in the case of small temperature gradients in a dilute suspension of nanoparticles [10]. It may be 
interesting to note that $R_{n}$ involves a different scaling (a typical nanofluid fraction rather than the difference of two fractions arising when constant but different nanoparticle volume fractions are imposed at the boundaries) due to the use of nanoparticle flux boundary conditions in the present study and hence it cannot be negative.

\subsection{Basic state}

The basic state is quiescent and is given by

$\vec{q}_{b}=0, p=p_{b}(z), T=T_{b}(z), \phi=\phi_{b}(z)$

where, the subscript $b$ denotes the basic state. Substituting Eq. (13) into Eqs. (7)-(10), we obtain

$\frac{d p_{b}}{d z}+R_{m} \hat{k}+R_{n} \phi_{b} \hat{k}-R_{t} T_{b} \hat{k}=0$

$\frac{d^{2} T_{b}}{d z^{2}}+\frac{N_{B}}{L e} \frac{d T_{b}}{d z} \frac{d \phi_{b}}{d z}+\frac{N_{A} N_{B}}{L e}\left(\frac{d T_{b}}{d z}\right)^{2}=0$

$N_{A} \frac{d^{2} T_{b}}{d z^{2}}+\frac{d^{2} \phi_{b}}{d z^{2}}=0$

A physically more realistic boundary condition on the nanoparticle volume fraction is considered as propounded by Nield and Kuznetsov [12]. That is, the nanoparticle flux is assumed to be zero rather than prescribing the volume fraction of nanoparticle on the impermeable boundaries.

Thus, we have

$$
\begin{array}{ll}
T_{b}=0, \frac{d \phi_{b}}{d z}+N_{A} \frac{d T_{b}}{d z}=0 & \text { at } z=0 \\
T_{b}=-1, \frac{d \phi_{b}}{d z}+N_{A} \frac{d T_{b}}{d z}=0 & \text { at } z=1 .
\end{array}
$$

Solving Eqs. (13) and (14) subject to the above boundary conditions, it is found that

$$
T_{b}=-z, \phi_{b}=\phi_{0}+N_{A} z
$$

where, $\phi_{0}$ is the reference value of nanoparticle volume fraction. From Eq. (17) it is observed that change in the boundary conditions on nanoparticle volume fraction amounts to basic temperature and volume fraction distributions to be remaining the same except for the 
coefficients. The pressure is of no consequence here as it will be eliminated subsequently.

\subsection{Perturbed state}

To study the stability of the basic state, we now superimpose perturbations on the basic state in the form

$\vec{q}=\vec{q}^{\prime}, p=p_{b}(z)+p^{\prime}, T=T_{b}(z)+T^{\prime}, \phi=\phi_{b}(z)+\phi^{\prime}$

where, $\vec{q}^{\prime}, p^{\prime}, T^{\prime}$ and $\phi^{\prime}$ are the perturbed quantities over their equilibrium counterparts and assumed to be small. Substituting Eq. (18) in Eqs. (7)-(10), linearizing and eliminating the pressure term from the momentum equation by operating curl twice and retaining the vertical component, we obtain the stability equations in the form

$$
\begin{aligned}
& \left(1+\Lambda_{1} \frac{\partial}{\partial t}\right)\left(R_{n} \nabla_{H}^{2} \phi^{\prime}-R_{t} \nabla_{H}^{2} T^{\prime}\right)=-\left(1+\Lambda_{2} \frac{\partial}{\partial t}\right) \nabla^{2} w^{\prime} \\
& M \frac{\partial T^{\prime}}{\partial t}-w^{\prime}=\nabla^{2} T^{\prime}-\frac{N_{A} N_{B}}{L e} \frac{\partial T^{\prime}}{\partial z}-\frac{N_{B}}{L e} \frac{\partial \phi^{\prime}}{\partial z} \\
& \frac{\partial \phi^{\prime}}{\partial t}+N_{A} w^{\prime}=\frac{N_{A}}{L e} \nabla^{2} T^{\prime}+\frac{1}{L e} \nabla^{2} \phi^{\prime} .
\end{aligned}
$$

It may be observed that Eqs. (20) and (21) are altered slightly due to change in the boundary conditions on volume fraction of nanoparticles [10].

The boundaries are impermeable and perfect conductors of heat. Hence, the boundary conditions are:

$$
\begin{array}{ll}
w^{\prime}=0, T^{\prime}=0, \frac{\partial \phi^{\prime}}{\partial z}+N_{A} \frac{\partial T^{\prime}}{\partial z}=0 & \text { at } z=0 \\
w^{\prime}=0, T^{\prime}=0, \frac{\partial \phi^{\prime}}{\partial z}+N_{A} \frac{\partial T^{\prime}}{\partial z}=0 & \text { at } z=1 .
\end{array}
$$

Assuming that the amplitudes of the perturbations are very small, we write $\left[w^{\prime}, T^{\prime}, \phi^{\prime}\right]=[W(z), \Theta(z), \Phi(z)] e^{\omega t+i(l x+m y)}$

where, $\omega\left(=\omega_{r}+i \omega_{i}\right)$ is the growth rate, $l$ and $m$ are the wave numbers in the $x$ and $y$ directions, respectively. Infinitesimal perturbations of the rest state may either decay or grow depending on the value of the parameter $\omega$.

Substituting Eq. (24) into Eqs. (19)-(21) and in the boundary conditions (22) and (23), we get 


$$
\begin{aligned}
& \left(D^{2}-a^{2}\right) W+a^{2} R_{t} \Theta-a^{2} R_{n} \Phi=\omega\left[-\Lambda_{2}\left(D^{2}-a^{2}\right) W-\Lambda_{1}\left(a^{2} R_{t} \Theta-a^{2} R_{n} \Phi\right)\right] \\
& W+\left(D^{2}-a^{2}-\frac{N_{A} N_{B}}{L e} D\right) \Theta-\frac{N_{B}}{L e} D \Phi=M \omega \Theta \\
& -N_{A} W+\frac{N_{A}}{L e}\left(D^{2}-a^{2}\right) \Theta+\frac{1}{L e}\left(D^{2}-a^{2}\right) \Phi=\omega \Phi
\end{aligned}
$$

and the boundary conditions become

$$
\begin{aligned}
& W=0, \Theta=0,\left(D \Phi+N_{A} D \Theta\right)=0 \text { at } z=0 \\
& W=0, \Theta=0,\left(D \Phi+N_{A} D \Theta\right)=0 \text { at } z=1
\end{aligned}
$$

where, $D=d / d z$.

\section{Numerical solution}

Equations (25)-(27) together with the boundary conditions (28) and (29) constitute a linear eigenvalue problem for the growth rate $\omega$ of the system. The resulting eigenvalue problem is solved numerically by the Galerkin method. Accordingly, the variables are written in a series of basis functions as

$$
W(z)=\sum_{i=1}^{N} A_{i} W_{i}(z), \quad \Theta(z)=\sum_{i=1}^{N} B_{i} \Theta_{i}(z), \quad \Phi(z)=\sum_{i=1}^{N} C_{i} \Phi_{i}(z)
$$

where $A_{i}, B_{i}$ and $C_{i}$ are unknown coefficients. The basis functions are represented by the power series satisfying the respective boundary conditions

$$
W_{i}(z)=\Theta_{i}(z)=z^{i}(1-z), \Phi_{i}(z)=N_{A} z^{i}(z-1)
$$

Multiplying Eq. (25) by $W_{j}(z)$, Eq. (26) by $\Theta_{j}(z)$ and Eq. (27) by $\Phi_{j}(z)$; performing the integration by parts with respect to $z$ between $z=0$ and 1 , and using the boundary conditions, we obtain the following system of algebraic equations:

$$
\begin{aligned}
& A_{i} C_{j i}+B_{i} D_{j i}+C_{i} E_{j i}=\omega\left\{A_{i} F_{j i}+B_{i} G_{j i}+C_{i} H_{j i}\right\} \\
& A_{i} I_{j i}+B_{i} J_{j i}+C_{i} K_{j i}=\omega B_{i} L_{j i} \\
& A_{i} M_{j i}+B_{i} N_{j i}+C_{i} P_{j i}=\omega C_{i} Q_{j i} .
\end{aligned}
$$


The coefficients $C_{j i}-Q_{j i}$ involve the inner products of the basis functions and are given by

$$
\begin{aligned}
& C_{j i}=-\left\{\left\langle D W_{j} D W_{i}\right\rangle+a^{2}\left\langle W_{j} W_{i}\right\rangle\right\}, D_{j i}=a^{2} R t\left\langle W_{j} \Theta_{i}\right\rangle, E_{j i}=-a^{2} R n\left\langle W_{j} \Phi_{i}\right\rangle, \\
& F_{j i}=\Lambda_{2}\left\{\left\langle D W_{j} D W_{i}\right\rangle+a^{2}\left\langle W_{j} W_{i}\right\rangle\right\}, G_{j i}=-\Lambda_{1} a^{2} R_{t}\left\langle W_{j} \Theta_{i}\right\rangle, H_{j i}=\Lambda_{1} a^{2} R_{n}\left\langle W_{j} \Phi_{i}\right\rangle, \\
& I_{j i}=\left\langle\Theta_{j} W_{i}\right\rangle, J_{j i}=-\left\langle D \Theta_{j} D \Theta_{i}\right\rangle-a^{2}\left\langle\Theta_{j} \Theta_{i}\right\rangle-\frac{N_{A} N_{B}}{L e}\left\langle\Theta_{j} D \Theta_{i}\right\rangle, K_{j i}=-\frac{N_{B}}{L e}\left\langle\Theta_{j} D \Phi_{i}\right\rangle, \\
& L_{j i}=M\left\langle\Theta_{j} \Theta_{i}\right\rangle, M_{j i}=-N_{A}\left\langle\Phi_{j} W_{i}\right\rangle, N_{j i}=-\frac{N_{A}}{L e}\left\{\left\langle D \Phi_{j} D \Theta_{i}\right\rangle+a^{2}\left\langle\Phi_{j} \Theta_{i}\right\rangle\right\} \\
& P_{j i}=-\frac{1}{L e}\left\{\left\langle D \Phi_{j} D \Phi_{i}\right\rangle+a^{2}\left\langle\Phi_{j} \Phi_{i}\right\rangle\right\}, Q_{j i}=\left\langle\Phi_{j} \Phi_{i}\right\rangle .
\end{aligned}
$$

The system of equations given by Eq. (32) is a generalized eigenvalue problem which can be written in the form

$A X=\omega B X$

where,

$$
A=\left(\begin{array}{ccc}
C_{j i} & D_{j i} & E_{j i} \\
I_{j i} & J_{j i} & K_{j i} \\
M_{j i} & N_{j i} & P_{j i}
\end{array}\right) ; \quad B=\left(\begin{array}{ccc}
F_{j i} & G_{j i} & H_{j i} \\
0 & L_{j i} & 0 \\
0 & 0 & Q_{j i}
\end{array}\right) \text { and } X=\left(\begin{array}{c}
A_{i} \\
B_{i} \\
C_{i}
\end{array}\right)
$$

We note that $A$ and $B$ are real matrices of order $N \times N$ and $X$ is the eigenvector. By using the subroutine GVLRG of the IMSL library, the complex eigenvalue $\omega$ is determined when the other parameters are specified. Then one of the parameters, say $R_{t}$, is varied until the real part of $\omega\left(\omega_{r}\right)$ vanishes. The zero crossing of $\omega_{r}$ is achieved by Newton's method for fixed point determination. The corresponding value of $R_{t}$ and $a$ are the critical conditions for neutral stability. Then the critical Rayleigh number with respect to the wave number is calculated using the golden section search method. The imaginary part of $\omega\left(\omega_{i}\right)$ indicates whether the instability onsets into steady convection or into growing oscillations. Thus the critical stability parameters $\left(R_{t c}, a_{c}, \omega_{i c}\right)$ are computed for different values of physical parameters involved therein. Convergence of the results is achieved by using six terms in the Galerkin expansion. 


\section{Results and Discussion}

The onset of convection in a horizontal layer of Darcy porous medium saturated with an Oldroyd-B viscoelastic nanofluid is investigated by considering a physically realistic boundary condition on volume fraction of nanoparticles. That is, the flux of volume fraction of nanoparticles is zero condition is used at the boundaries. The resulting eigenvalue problem is solved numerically by employing Galerkin method. The parametric values vary with the base fluid and nanoparticles chosen. The ratio of density of the nanoparticles to that of a base fluid for $\mathrm{Cu}$ (copper) and $\mathrm{Ag}$ (silver) is 8.96 and 10.5, respectively. The ratio of heat capacity based on the volume fraction of nanoparticles to that of a base fluid is 0.83 for $\mathrm{Cu}$ and 0.59 for Ag. So, by following Nield and Kuznetsov [12], Tzou [6, 7] and Buongiorno [8] we have taken the values of concentration Darcy-Rayleigh number $R_{n}$ in the order $1 \sim 10$, modified particle density increment $N_{B}$ is of the order $10^{-3} \sim 10^{-1}$ and Lewis number $L e$ is taken in the order of $1 \sim 10$. The value of modified diffusivity ratio $N_{A}$ is not more than 10 .

To have a check on the accuracy of the numerical procedure used, first test computations are carried out for regular fluid (i.e. $N_{A}=0$ ) for different values of strain retardation parameter $\Lambda_{2}$ by fixing the stress relaxation parameter, $\Lambda_{1}$ and the heat capacity ratio, $M$ at unity. For regular fluids, exact solution can readily be obtained with $W=\sin \pi z=$ $\Theta$ as the solution for the eigenvalue problem and the critical stability parameters are found to be

$$
R_{t c}=\frac{\left(\pi^{2}+a_{c}^{2}\right)\left\{M+\left(\pi^{2}+a_{c}^{2}\right) \Lambda_{2}\right\}}{a_{c}^{2} \Lambda_{1}} ; a_{c}^{2}=\pi \sqrt{\pi^{2}+\frac{M}{\Lambda_{2}}} ; \omega_{i c}^{2}=\frac{\left(\pi^{2}+a_{c}^{2}\right)\left(\Lambda_{1}-\Lambda_{2}\right)-M}{\Lambda_{1} \Lambda_{2} M}
$$

In Table 1, the results computed for different orders of approximations in the Galerkin expansion are compared with those obtained from Eq. (34). It is noted that the exact results and those obtained by considering six terms in the Galerkin expansion complement with each other indicating the accuracy of the numerical procedure used. Table 2 shows the numerically computed triplets $\left(R_{t c}, a_{c}, \omega_{i c}\right)$ at different orders of approximation in the Galerkin expansion for various values of $\Lambda_{2}$ for the nanofluid case with $N_{A}=2, R_{n}=1$, $N_{B}=0.01, \Lambda_{1}=1, L e=1$ and $M=1$. From the tabulated values, again it is observed that the results converge for six terms in the Galerkin expansion. Hence, the results are presented by taking six terms in the Galerkin expansion. 
Figures 2 (a-f) show the neutral stability curves in the $\left(R_{t}, a\right)$-plane for different values of stress relaxation parameter $\Lambda_{1}$ (Fig. 2a), strain retardation parameter $\Lambda_{2}$ (Fig. 2b), modified diffusivity ratio $N_{A}$ (Fig. 2c), concentration Darcy-Rayleigh number $R_{n}$ (Fig. 2d), Lewis number $L e$ (Fig. 2e) and heat capacity ratio $M$ (Fig. 2f). The plots shown in Fig. 2(a) and (b) are for different values of $\Lambda_{1}$ and $\Lambda_{2}$, respectively. The figures reveal that oscillatory convection is possible only if $\Lambda_{2}<\Lambda_{1}$ as observed in the case of regular fluids. Moreover, $\Lambda_{1}$ and $\Lambda_{2}$ have opposing contributions on the stability of the system. That is, increasing $\Lambda_{1}$ is to decrease the region of stability, while the effect of $\Lambda_{2}$ is to increase the region of stability. Besides, there exists a critical value of $\Lambda_{1}(=0.15)$ above which oscillatory convection is possible and below which stationary convection occurs (Fig. 2a). To the contrary, there exists a critical value of $\Lambda_{2}(=0.945)$ below which oscillatory convection is possible and above which stationary convection occurs for the parametric values chosen (Fig. 2b). That is, oscillatory convection occurs for comparatively stronger elastic fluids at thermal Darcy-Rayleigh numbers lower than that of stationary convection. From Figs. 2(c), (d) and (e) it is observed that oscillatory convection is predicted for all wave numbers and increasing $N_{A}, R_{n}$ and $L e$ is to decrease the region of stability. It may also be noted that the oscillatory neutral curves for different values of Le coalesce at lower wave number region. Increase in $M$ is to increase only the oscillatory thermal Darcy-Rayleigh number and hence its effect is to increase the region of stability (Fig. 2f). Due to consideration of the changed boundary conditions on volume fraction of nanoparticles, instability is found to appear in nonoscillatory form in the absence of viscoelastic effects.

The variation of critical thermal Darcy-Rayleigh number $R_{t c}$, the corresponding critical wave number $a_{c}$ and the critical frequency of oscillations $\omega_{i c}$ as a function $\Lambda_{2}$ is displayed in Figs. 3-8 for various values of physical parameters to know their influence on the stability characteristics of the system. In general, the elasticity parameters $\Lambda_{1}$ and $\Lambda_{2}$ have no influence on the onset of stationary convection. Thus, there is no distinction between the Newtonian nanofluid and viscoelastic nanofluid as far as stationary convection is concerned. This is because the basic state remains the same for both Newtonian and viscoelastic nanofluids as it corresponds to pure conduction. The curves of $\omega_{i c}$ are ending at points beyond which the frequency of oscillations is not positive. 
Figure 3 shows the results for different values of $\Lambda_{1}$ when $R_{n}=1, L e=1, N_{A}=2$, $N_{B}=0.01$ and $M=1$. The effect of increasing $\Lambda_{1}$ is to decrease the critical oscillatory Darcy-Rayleigh number for a fixed value of $\Lambda_{2}$ and therefore its effect is to hasten the onset of oscillatory convection. This may be attributed to the fact that the relaxation time reduces the shear rate (i.e., increases the elasticity of a viscoelastic fluid) thus causing instability. An opposite trend could be seen with increasing $\Lambda_{2}$. Moreover, the ranges of values of $\Lambda_{2}$ within which oscillatory convection is possible increase with increasing $\Lambda_{1}$. In other words, for a fixed value of $\Lambda_{1}$, there exists a value $\Lambda_{2}^{c}$ which divides the boundary of regimes between oscillatory and stationary convection. Initially, the convection begins in the form of an oscillatory mode, and as the value of $\Lambda_{2}$ reaches $\Lambda_{2}^{c}$, convection ceases to be oscillatory, and stationary convection becomes the preferred mode of instability. The value of $\Lambda_{2}^{c}$ depends on other physical parameters as well. The critical wave number $a_{c}$ (Fig. 3b) and critical frequency of oscillations $\omega_{i c}$ (Fig. 3c) decrease with increasing $\Lambda_{2}$. The curves of $a_{c}$ drop suddenly as the transition from oscillatory to stationary convection occurs at different values of $\Lambda_{2}$ and in the figure the vertical lines represent this transition. Furthermore, there is no noticeable change in $a_{c}$ with increasing $\Lambda_{1}$. The values of critical frequency of oscillations $\omega_{i c}$ increase with increasing $\Lambda_{1}$ due to an increase in the elasticity of the fluid and the same is evident from Fig. 3(c).

The effect of ratio of heat capacities $M$ is presented in Fig. 4 and it affects only the onset of oscillatory convection. Increasing $M$ is to delay the onset of oscillatory convection and to decrease the ranges of values of $\Lambda_{2}$ within which oscillatory convection is possible (Fig. 4a). The critical wave number increases with increasing $M$ (Fig. 4b) but the critical frequency decreases (Fig. 4c).

The effect of modified diffusivity ratio $N_{A}$ is presented in Fig. 5 for two values of stress relaxation parameter $\Lambda_{1}(=0.25$ and 0.3$)$ when $R_{n}=1, L e=1, M=1$ and $N_{B}=0.01$. In Fig. 5(a), the curves lying to the left of the discontinuity in slope (shown by dotted lines) correspond to oscillatory onset, while to the right the onset is of stationary type (continuous line). The curves for $N_{A}=0$ correspond to the case of regular non-Newtonian fluid and they 
lie above all other curves of $N_{A} \neq 0$. Thus the effect of increasing $N_{A}$ is to hasten the onset of oscillatory and stationary convection nearly to the same extent. That is thermophoresis dominates over the Brownian diffusion to diffuse the nanoparticles as a result increase in $N_{A}$ hastens the onset. The effect of increasing $\Lambda_{2}$ is to delay the onset of oscillatory convection and opposite is the case with increasing $\Lambda_{1}$. The value of $\Lambda_{2}$, at which the preferred mode of instability from oscillatory to stationary convection changes, decreases with increasing $N_{A}$ and increases with increasing $\Lambda_{1}$. Figures 5(b) and (c) show the variation of critical wave number $a_{c}$ and critical frequency $\omega_{i c}$ for $\Lambda_{1}=0.25$. It is observed that, increase in $N_{A}$ is to increase $a_{c}$ marginally in the case of oscillatory onset but the curves of different $N_{A}$ come together with increasing $\Lambda_{2}$ before showing an opposite trend in the case of stationary onset. The critical frequency of oscillations $\omega_{i c}$ decreases with increasing $N_{A}$ (Fig. 5c).

Figure 6 illustrates the results for different values of concentration Darcy-Rayleigh number $R_{n}$ when $N_{A}=1, L e=1, M=1$ and $N_{B}=0.01$ for two values of $\Lambda_{1}(=0.25$ and 0.3$)$. From Fig. 6(a) it is noted that the onset of convective instability is hastened with increasing concentration Darcy Rayleigh number $R_{n}$. This is because increase in the density of nanoparticles is to enhance the heat transfer and hence to advance the onset of convection. Also, increase in the value of $R_{n}$ and decrease in the value of $\Lambda_{1}$ is to decrease the threshold value of $\Lambda_{2}$ at which the preferred mode of instability changes. The critical wave number increases marginally with an increase in the value of $R_{n}$ but the curves of different $R_{n}$ come together with increasing $\Lambda_{2}$ before showing an opposite trend in the case of stationary onset (Fig. 6b). The critical frequency of oscillation decreases with increasing $R_{n}$ (Fig. 6c).

The effect of modified particle density increment $N_{B}$ is shown in Fig. 7 for two values of $\Lambda_{1}(=0.28$ and 0.3$)$ when $N_{A}=2, R_{n}=2, M=1$ and $L e=1$. From Fig. 7(a), it is seen that an increase in the value of $N_{B}$ is to hasten the oscillatory and stationary onset. This is because the heavier nanoparticles moving through the base fluid makes stronger disturbances as compared with the lighter nanoparticles which in turn produce more heat transfer in the fluid. However, the effect of $N_{B}$ on the oscillatory onset is found to be not so strong compared to its influence on the stationary onset. Also, there is no considerable 
change in the value of $\Lambda_{2}$ at which the preferred mode of instability changes. The variation in the critical wave number with $N_{B}$ is insignificant in the case of oscillatory onset, while in the case of stationary onset increasing $N_{B}$ is to decrease $a_{c}$ slightly (Fig.7b). Figure 7 (c) shows that increasing $N_{B}$ is to decrease $\omega_{i c}$ marginally.

Figure 8 demonstrates the effect of Lewis number $L e$ for two values of $\Lambda_{1}(=0.8$ and 1) when $N_{A}=1, R_{n}=2, M=1$ and $N_{B}=0.01$. As seen from Fig. 8(a), increase in Le hastens the onset of oscillatory and stationary convection as thermal diffusion is dominated over Brownian diffusion. From the figure it is also noted that the influence of $L e$ on the onset of stationary convection is more pronounced than on the oscillatory convection. The value of $\Lambda_{2}$ at which the preferred mode of instability changes decreases significantly with an increase in the value of $L e$ and decrease in the value of $\Lambda_{1}$. In the case of stationary convection, the critical wave number decreases with increasing Le but an opposite trend is noticed in the case of oscillatory convection (Fig. 8b). The critical frequency decreases with increasing $L e$ (Fig. 8c). Since the effect of $\Lambda_{1}$ found to be insignificant on $a_{c}$ and $\omega_{i c}$ with increasing $N_{A}, R_{n}, N_{B}$ and $L e$, its effect is not shown in the respective figures.

\section{Conclusions}

The onset of convection in a horizontal layer of modified Darcy porous medium saturated with an Oldroyd-B viscoelastic nanofluid is investigated by considering flux of volume fraction of nanoparticles is zero on the boundaries; a physically realistic boundary condition. The generalized eigenvalue problem is solved numerically using the Galerkin method.

The results of the forgoing study may be summarized as follows:

(i) The oscillatory convection is possible only if the strain retardation parameter $\Lambda_{2}$ is less than the stress relaxation parameter $\Lambda_{1}$ and also when $\Lambda_{2}$ is less than a threshold value which in turn depends on the other parametric values. The parameters $\Lambda_{1}$ and $\Lambda_{2}$ have opposing contributions on the onset of oscillatory 
convection. Increase in the value of $\Lambda_{2}$, ratio of heat capacities $M$ is to delay the onset of oscillatory convection.

(ii) The effect of increasing modified diffusivity ratio $N_{A}$, concentration DarcyRayleigh number $R_{n}$, modified particle density increment $N_{B}$ and Lewis number $L e$ is to hasten the onset of both oscillatory and stationary convection. The effect $N_{B}$ and Le on oscillatory convection is not so significant compared to their influence on the stationary onset.

(iii) The range of $\Lambda_{2}$ within which oscillatory convection is preferred gets decreased with increasing $N_{A}, R_{n}, N_{B}, L e$ and $M$. In contrast to this, the range of $\Lambda_{2}$ increases with increasing $\Lambda_{1}$.

(iv) Increase in the value of $\Lambda_{2}$ is to increase the size of convection cells of oscillatory onset and this trend gets reversed with increasing $\Lambda_{1}$ and $M$. Also, the effect of increasing $N_{A}, R_{n}$ and $L e$ is to decrease the size of convection cells of oscillatory onset but opposite is the case with the stationary onset. The variation in the critical wave number $a_{c}$ with $N_{B}$ is insignificant in the case of oscillatory onset, while $a_{c}$ decreases in the case of stationary onset with increasing $N_{B}$.

(v) The values of critical wave number for the oscillatory onset are higher than those of stationary onset.

(vi) The critical frequency of oscillations $\omega_{i c}$ decreases with increasing $N_{A}, R_{n}, M$, $N_{B}$, Le and $\Lambda_{2}$. To the contrary, increase in the value of $\Lambda_{1}$ is to increase the value of $\omega_{i c}$.

\section{Acknowledgement}

One of the authors (M.D) wishes to thank the Principal and the Management of his college for their encouragement and support. The authors wish to thank the reviewers for their useful suggestions.

\section{References}

[1] S. Choi, Enhancing thermal conductivity of fluids with nanoparticles. In: Siginer D.A., Wang, H.P. (eds.) Developments and Applications of Non-Newtonian Flows, ASME FED-Vol. 231/MD- ASME, New York 66 (1995) 99-105. 
[2] S. Kakac, A. Pramuanjaroenkij, Review of convective heat transfer enhancement with nanofluids, Int. J. Heat Mass Trans. 52 (2009) 3187-3196.

[3] W. Yu, H. Xie, A review on nanofluids: Preparation, stability mechanisms, and applications, J. Nanomaterials (2012) 1-17.

[4] E.K. Goharshadi, H. Ahmadzadeh, S. Samiee, M. Hadadian, Nanofluids for heat transfer enhancement - A review, Phys. Chem. Res. 1 (2013) 1-33.

[5] I.M. Mahbubul, R. Saidur, M. A. Amalina, Latest developments on the viscosity of nanofluids, Int. J. Heat Mass Trans. 55 (2012) 874-885.

[6] D. Y. Tzou, Instability of nanofluids in natural convection, ASME J. Heat Trans. 130 (2008) 072401.

[7] D. Y. Tzou, Thermal instability of nanofluids in natural convection, Int. J. Heat Mass Trans. 51 (2008) 2967-2979.

[8] J. Buongiorno, Convective transport in nanofluids, ASME J. Heat Transf. 128 (2006) 240-250.

[9] J. Kim, Y. T. Kang, C. K. Choi, Analysis of convective instability and heat transfer characteristics of nanofluids, Phys. Fluids 16 (2004) 2395-2401.

[10] D.A. Nield, A.V. Kuznetsov, Thermal instability in a porous medium layer saturated by a nanofluid, Int. J. Heat Mass Trans. 52 (2009) 5796-5801.

[11] D.A. Nield, A. Bejan, Convection in Porous Media, 4th ed., Springer, New York, 2013.

[12] D.A. Nield, A.V. Kuznetsov, Thermal instability in a porous medium layer saturated by a nanofluid: A revised model, Int. J. Heat Mass Trans. 68 (2014) 211-214.

[13] P. Namburu, D. Kulkarni, D. Misra, D. Das, Viscosity of copper oxide nanoparticles dispersed in ethylene glycol and water mixture, Exp. Therm. Fluid Sci. 32 (2) (2007) 397-402.

[14] H.S. Chen, Y.L. Ding, C.Q. Tan, Rheological behaviour of nanofluids, New J. Phys. 9 (2007) 367.

[15] H.S. Chen, Y.L. Ding, Y.R. He, C.Q. Tan, Rheological behaviour of ethylene glycol based titania nanofluids, Chem. Phys. Lett. 444 (2007) 333-337.

[16] H.S. Chen, Y. Ding, A. Lapkin, Rheological behaviour of nanofluids containing tube/rod-like nanoparticles, Power Technol. 194 (2009) 132-141.

[17] A.J. Schmidt, M. Chiesa, D.H. Torchinsky, J.A. Johnson, A. Boustani, G.H. McKinley, K.A. Nelson, G. Chen, Experimental investigation of nanofluid shear and longitudinal viscosities, Appl. Phys. Lett. 92 (2008) 244107. 
[18] R. B. Bird, R. C. Armstrong, O. Hassager, Dynamics of polymeric liquids, $2^{\text {nd }}$ ed., John Wiley \& Sons, 1987.

[19] Z. Li, R.E. Khayat, Finite amplitude Rayleigh-Bénard convection and pattern selection for viscoelastic fluids, J. Fluids Mech. 529 (2005) 221-251.

[20] R. I. Tanner, Engineering Rheology, Oxford University press, Oxford, 1985.

[21] R.G. Larson, Constitutive Equations for polymer melts and solutions, Butterworths, 1988.

[22] M.G. Alishaev, A. K. Mirzadjanzade, For the calculation of delay phenomenon in filtration theory, Izv Vuzov Neft I Gas (Russ Chem Rev) 6 (1975) 71-77.

[23] N. Rudraiah, P.N. Kaloni, P.V. Radhadevi, Oscillatory convection in a viscoelastic fluid through a porous layer heated from below, Rheol. Acta 28 (1989) 48-52.

[24] A. V. Shenoy, Non-Newtonian fluid heat transfer in porous media, Adv. Heat Trans. 24 (1994) 90-101.

[25] M.C. Kim, S.B. Lee, B.J. Chung, Thermal instability of viscoelastic fluids in porous media, Int. J. Heat Mass Trans. 46 (2003) 5065-5072.

[26] M.S. Malashetty, I.S. Shivakumara, S. Kulkarni, M. Swamy, Convective instability of Oldroyd B fluid saturated porous layer heated from below using a thermal nonequilibrium model, Transp. Porous Med. 64 (2006) 123-139.

[27] I.S. Shivakumara, M.S. Malashetty, K.B. Chavaraddi, Onset of convection in a viscoelastic-fluid-saturated sparsely packed porous layer using a thermal nonequillibrium model, Can. J. Phys. 84 (2006) 973-990.

[28] Z. Zhang, C. Fu, W. Tan, Linear and nonlinear stability analyses of thermal convection for Oldroyd-B fluids in porous media heated from below, Phys. Fluids 20 (2008) 084103-1-12

[29] L.J. Sheu, Thermal instability in a porous medium layer saturated with a viscoelastic nanofluid, Transp. Porous Med. 88 (2011) 461-477.

[30] Yadav, D., Bhargava, R., Agrawal, G.S., Yadav, N., Lee, J., Kim, M.C.: Linear and nonlinear analysis of thermal instability in a rotating porous layer saturated by a nonNewtonian nanofluid with thermal conductivity and viscosity variation, Microfluid Nanofluid. 16 (2014) 425-440. 
Table 1: Comparison of $R_{t c}, a_{c}$ and $\omega_{i c}$ for different orders of approximations in the Galerkin expansion for regular fluids $\left(N_{A}=0\right)$ with $M=1$

\begin{tabular}{|l|c|c|c|c|c|c|c|c|c|}
\hline \multirow{2}{*}{$N$} & \multicolumn{3}{|c|}{$\Lambda_{2}=0.1$} & \multicolumn{3}{c|}{$\Lambda_{2}=0.3$} & \multicolumn{3}{c|}{$\Lambda_{2}=0.5$} \\
\cline { 2 - 10 } & $R_{t c}$ & $a_{c}$ & $\omega_{i c}$ & $R_{t c}$ & $a_{c}$ & $\omega_{i c}$ & $R_{t c}$ & $a_{c}$ & $\omega_{i c}$ \\
\hline 1 & 5.8284 & 3.760 & 14.3958 & 13.9282 & 3.398 & 6.8514 & 21.9544 & 3.310 & 4.3539 \\
\hline 2 & 5.8284 & 3.760 & 14.3958 & 13.9282 & 3.398 & 6.8514 & 21.9544 & 3.310 & 4.3539 \\
\hline 3 & 5.7747 & 3.742 & 14.3126 & 13.7711 & 3.379 & 6.8071 & 21.6934 & 3.290 & 4.3236 \\
\hline 4 & 5.7747 & 3.742 & 14.3126 & 13.7711 & 3.379 & 6.8071 & 21.6934 & 3.290 & 4.3236 \\
\hline 5 & 5.7747 & 3.741 & 14.3102 & 13.7709 & 3.378 & 6.8060 & 21.6931 & 3.290 & 4.3236 \\
\hline 6 & 5.7747 & 3.741 & 14.3102 & 13.7709 & 3.378 & 6.8060 & 21.6931 & 3.290 & 4.3236 \\
\hline $\begin{array}{l}\text { Exact } \\
\text { solution }\end{array}$ & 5.7747 & 3.742 & 14.3120 & 13.770 & 3.378 & 6.8067 & 21.6931 & 3.290 & 4.3236 \\
\hline
\end{tabular}

Table 2: Comparison of $R_{t c}, a_{c}$ and $\omega_{i c}$ for different orders of approximations in the Galerkin expansion for nanofluids with $N_{A}=2, R_{n}=1, N_{B}=0.01, \Lambda_{1}=1, L e=1$ and $M=1$.

\begin{tabular}{|l|c|c|c|c|c|c|c|c|c|}
\hline \multirow{3}{*}{$N$} & \multicolumn{3}{|c|}{$\Lambda_{2}=0.1$} & \multicolumn{3}{c|}{$\Lambda_{2}=0.3$} & \multicolumn{3}{c|}{$\Lambda_{2}=0.5$} \\
\cline { 2 - 10 } & $R_{t c}$ & $a_{c}$ & $\omega_{i c}$ & $R_{t c}$ & $a_{c}$ & $\omega_{i c}$ & $R_{t c}$ & $a_{c}$ & $\omega_{i c}$ \\
\hline 1 & 2.2946 & 3.8990 & 12.5048 & 10.1734 & 3.426 & 6.4023 & 18.1214 & 3.3220 & 4.1570 \\
\hline 2 & 2.1915 & 3.8940 & 11.8231 & 9.8819 & 3.383 & 6.0665 & 17.7115 & 3.2730 & 3.9652 \\
\hline 3 & 2.1644 & 3.8810 & 11.7836 & 9.7603 & 3.367 & 6.0374 & 17.4911 & 3.2570 & 3.9440 \\
\hline 4 & 2.1300 & 3.8770 & 11.7471 & 9.7206 & 3.366 & 6.0297 & 17.4497 & 3.2560 & 3.9403 \\
\hline 5 & 2.1296 & 3.8760 & 11.7447 & 9.7203 & 3.366 & 6.0297 & 17.4493 & 3.2560 & 3.9403 \\
\hline 6 & 2.1296 & 3.8770 & 11.7470 & 9.7203 & 3.366 & 6.0297 & 17.4493 & 3.2560 & 3.9403 \\
\hline
\end{tabular}




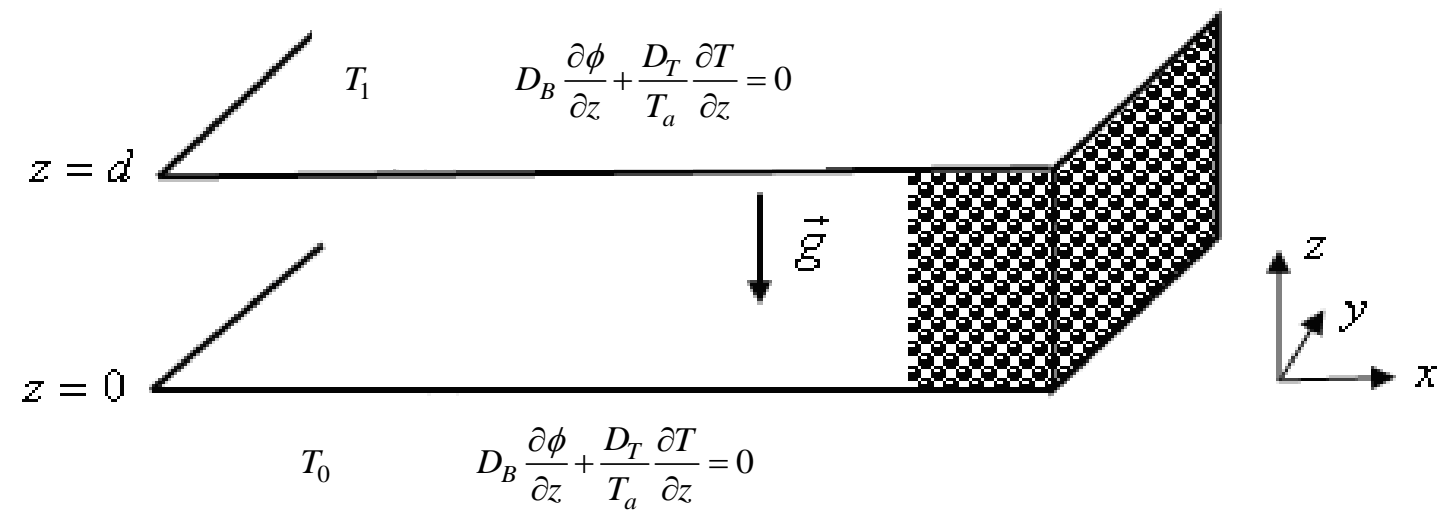

Fig. 1 Physical configuration 

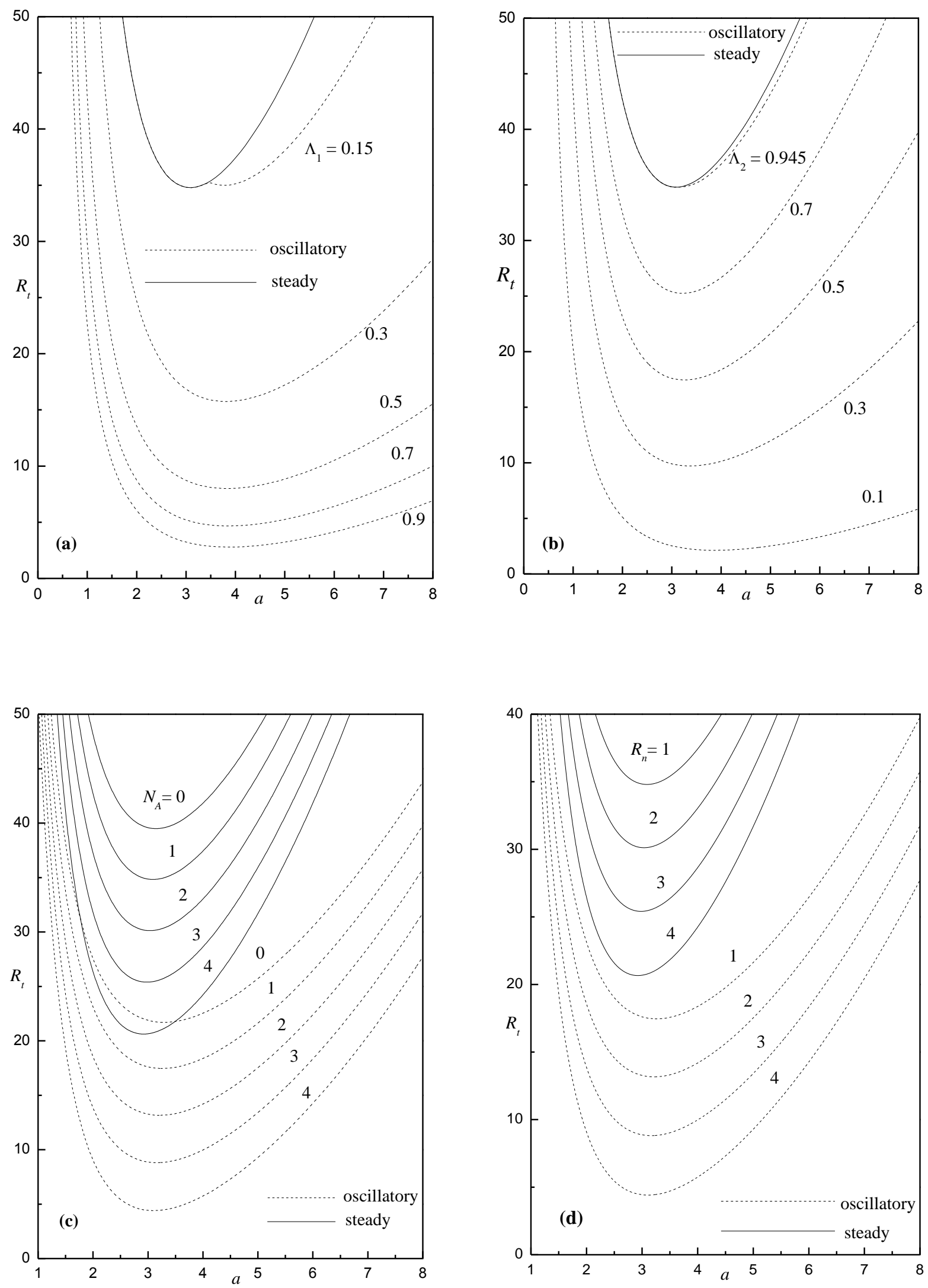

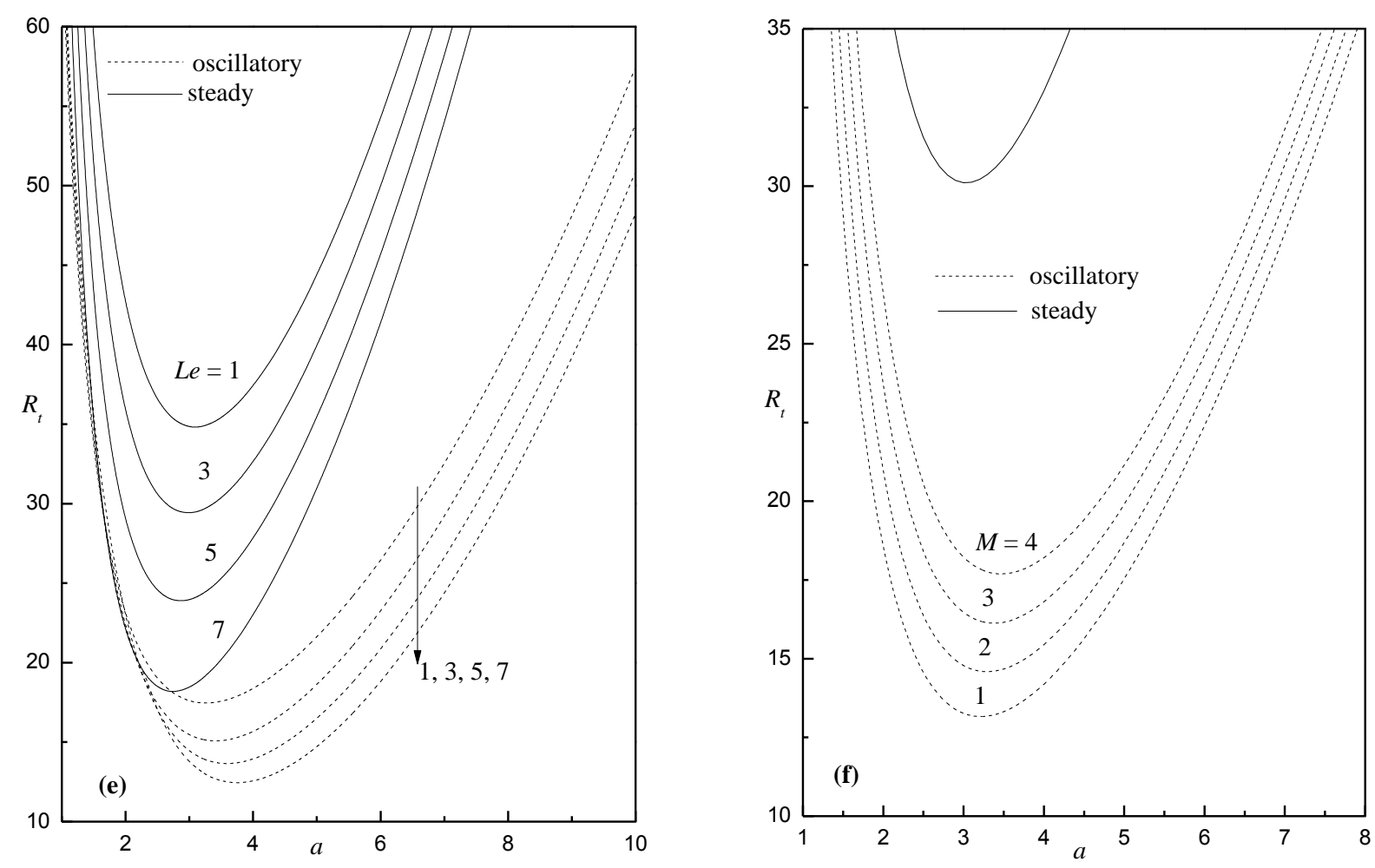

Fig. 2: Neutral curves for different values of (a) $\Lambda_{1}$ with $N_{A}=2, R_{n}=1, N_{B}=0.01, \Lambda_{2}=0.1$, $L e=1$ and $M=1$ (b) $\Lambda_{2}$ with $N_{A}=2, R_{n}=1, N_{B}=0.01, \Lambda_{1}=1, L e=1$ and $M=1$ (c) $N_{A}$ with $R_{n}=2, N_{B}=0.01, L e=1, \Lambda_{1}=1, \Lambda_{2}=0.5$ and $M=1$ (d) $R_{n}$ with $N_{A}=2, N_{B}=0.01$ $L e=1, \Lambda_{1}=1, \Lambda_{2}=0.5$ and $M=1$ (e) $L e$ with $R_{n}=2, N_{A}=1, N_{B}=0.01, \Lambda_{1}=1, \Lambda_{2}=0.5$ and $M=1$ (f) $M$ with $L e=1, R_{n}=2, N_{A}=2, N_{B}=0.01, \Lambda_{1}=1$ and $\Lambda_{2}=0.5$. 

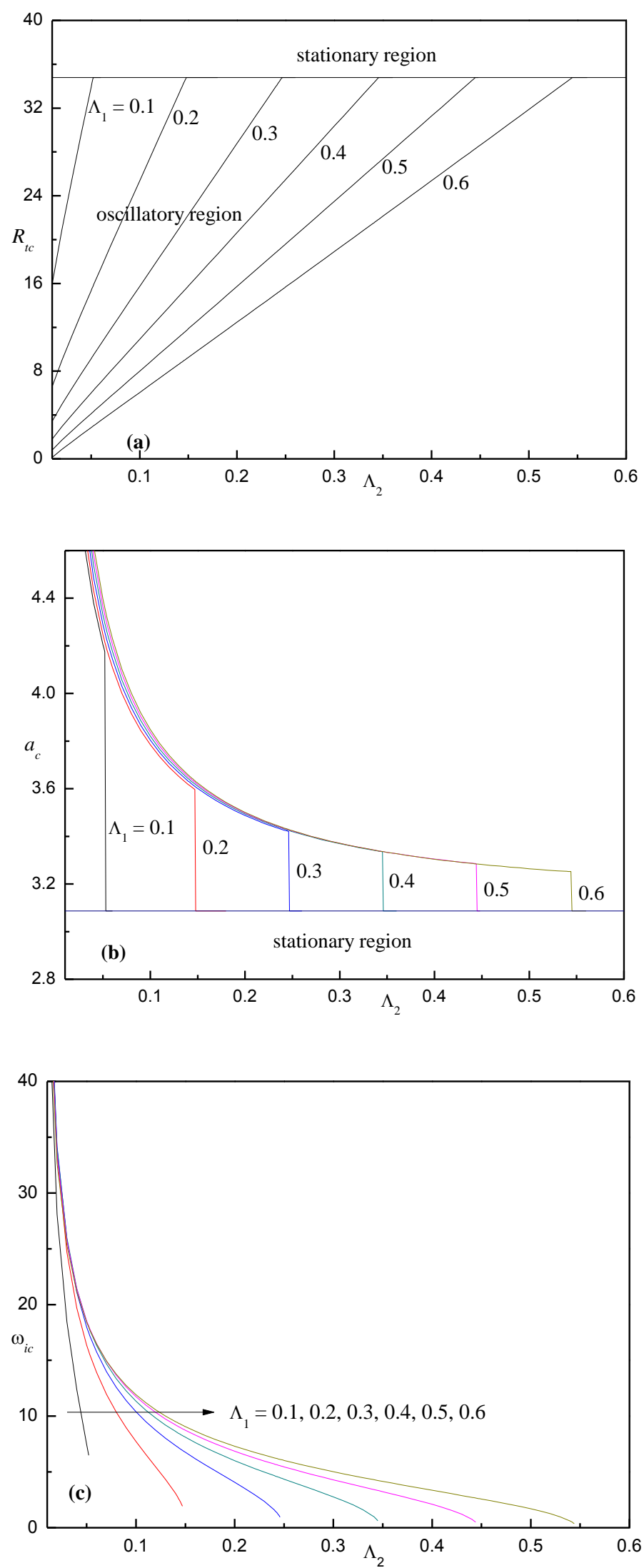

Fig. 3 Variation of (a) $R_{t c}$ (b) $a_{c}$ (c) $\omega_{i c}$ with $\Lambda_{2}$ for different values of $\Lambda_{1}$ when $R_{n}=1$, $L e=1, N_{A}=2, N_{B}=0.01$ and $M=1$. 

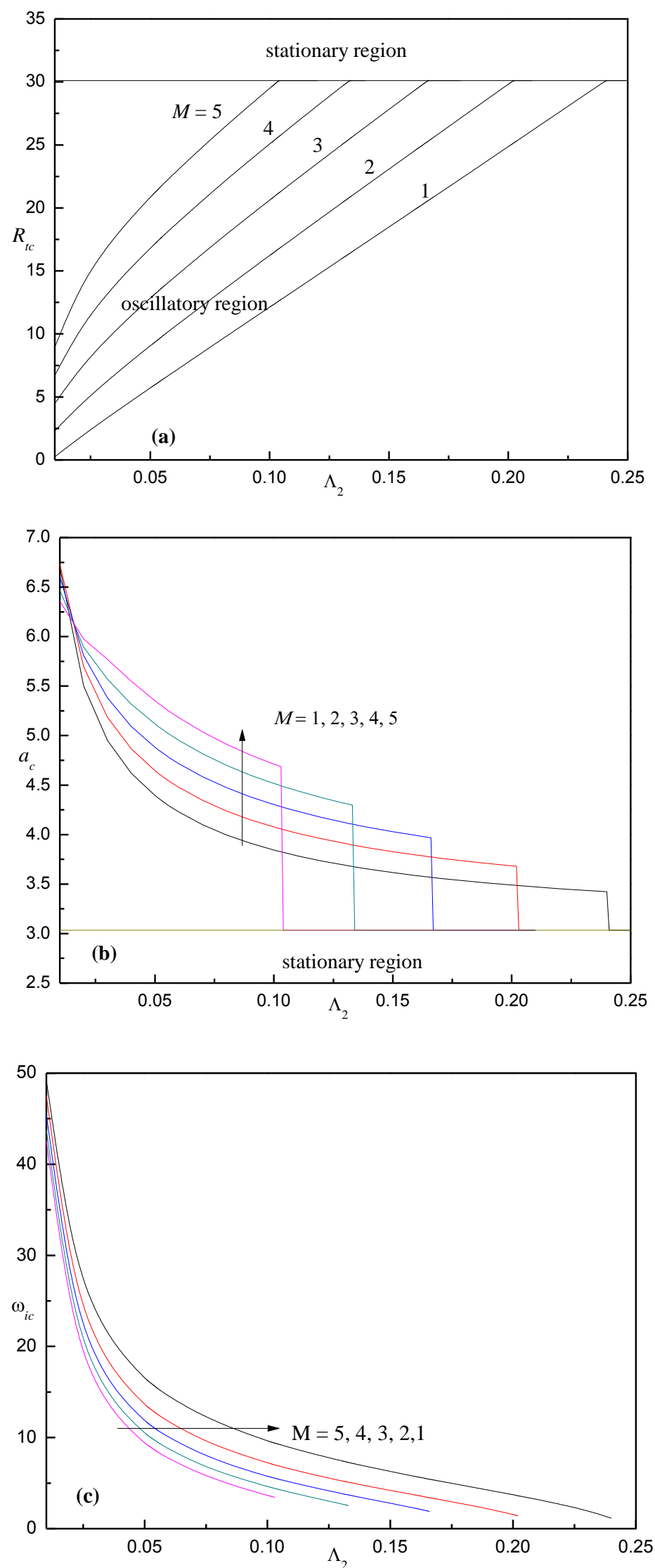

Fig. 4 Variation of (a) $R_{t c}$ (b) $a_{c}$ (c) $\omega_{i c}$ with $\Lambda_{2}$ for different values of $M$ when $R_{n}=2$, $L e=1, N_{A}=2, N_{B}=0.01$ and $\Lambda_{1}=0.3$. 

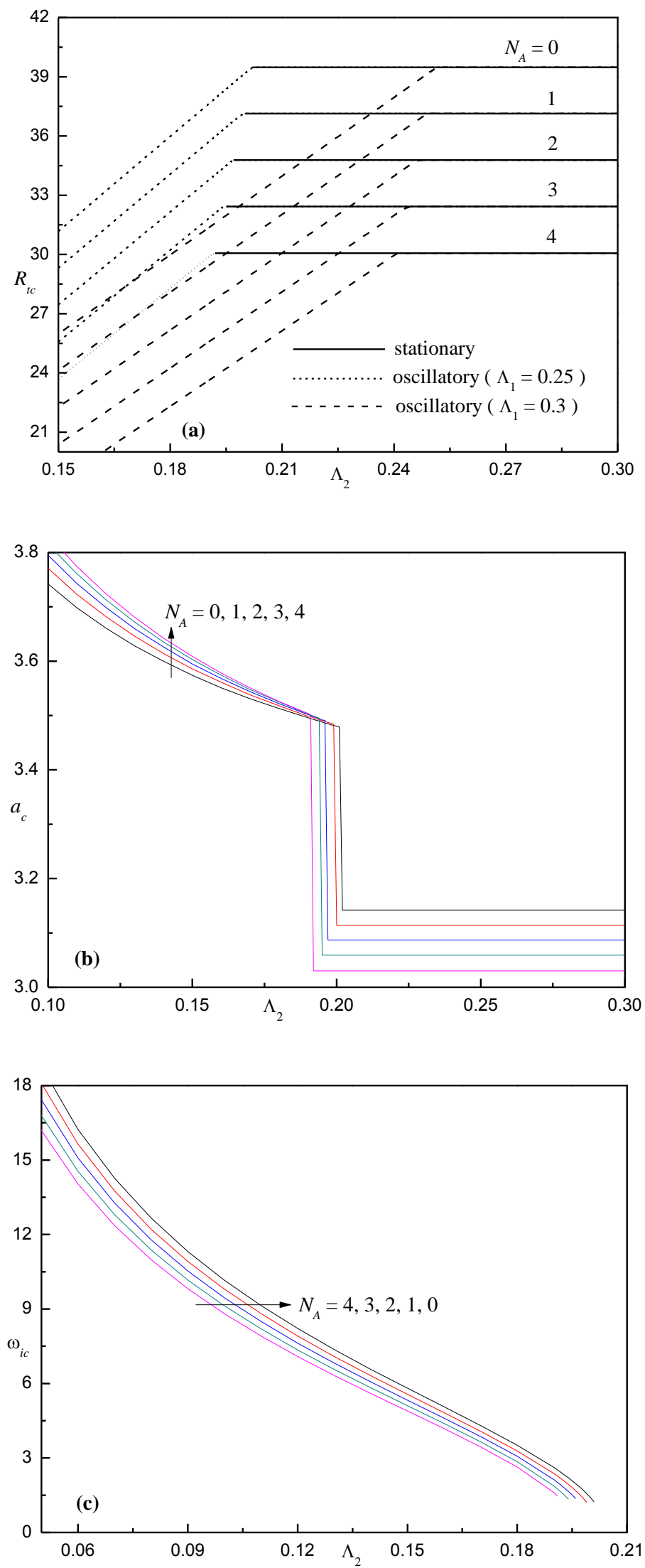

Fig. 5 Variation of (a) $R_{t c}$ (b) $a_{c}\left(\Lambda_{1}=0.25\right)$ (c) $\omega_{i c}\left(\Lambda_{1}=0.25\right)$ with $\Lambda_{2}$ for different values of $N_{A}$ when $R_{n}=1, L e=1, M=1$ and $N_{B}=0.01$. 

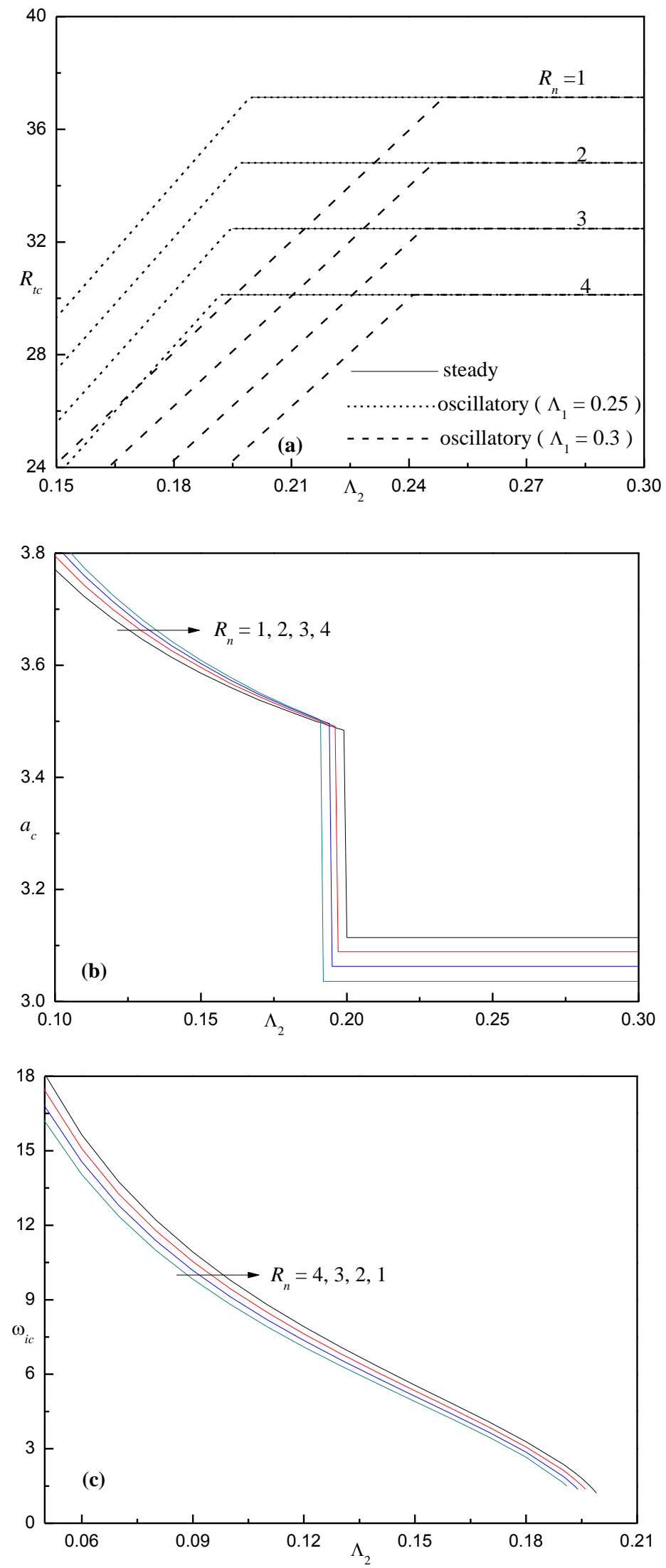

Fig. 6 Variation of (a) $R_{t c}$ (b) $a_{c}\left(\Lambda_{1}=0.25\right)$ (c) $\omega_{i c}\left(\Lambda_{1}=0.25\right)$ with $\Lambda_{2}$ for different values of $R_{n}$ when $N_{A}=1, L e=1, M=1$ and $N_{B}=0.01$. 

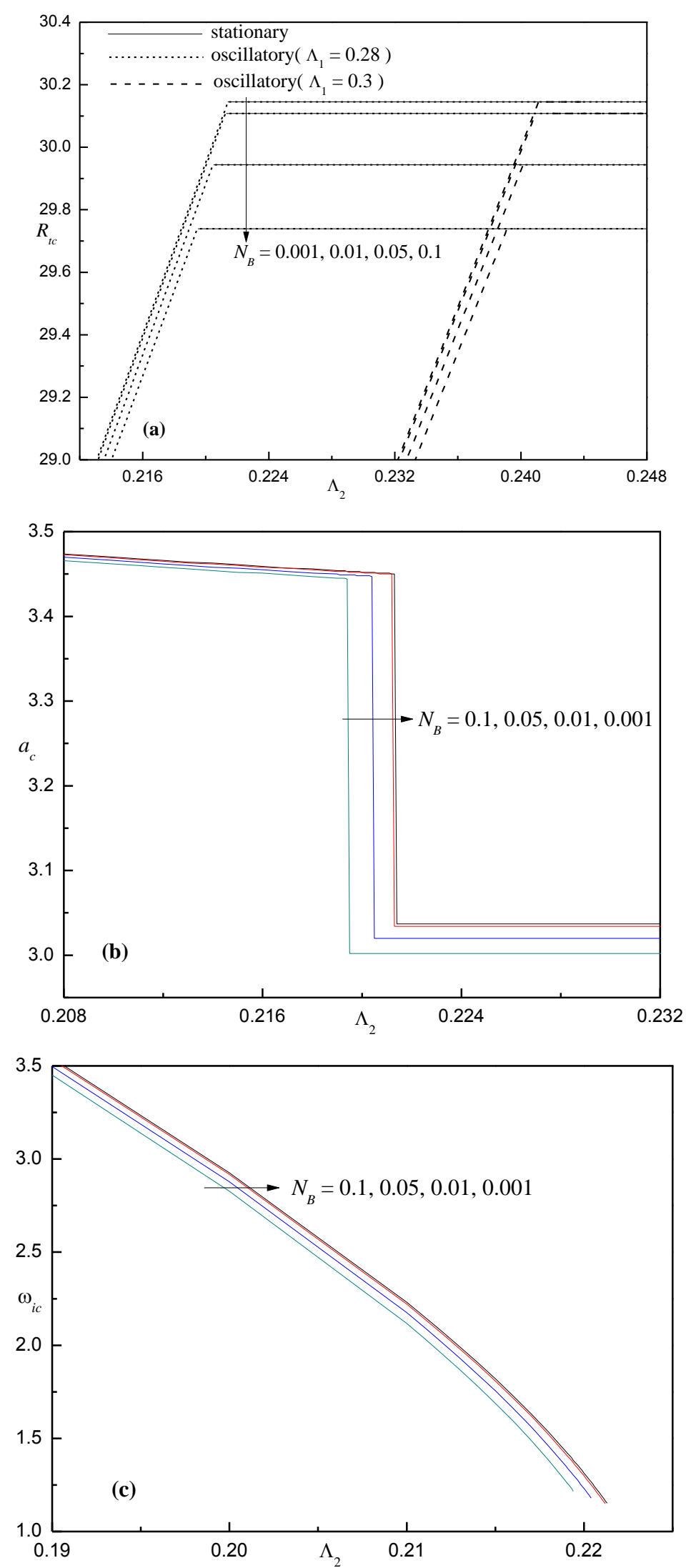

Fig. 7 Variation of (a) $R_{t c}$ (b) $a_{c}\left(\Lambda_{1}=0.28\right)$ (c) $\omega_{i c}\left(\Lambda_{1}=0.28\right)$ with $\Lambda_{2}$ for different values of $N_{B}$ when $N_{A}=2, R_{n}=2, M=1, L e=1$. 

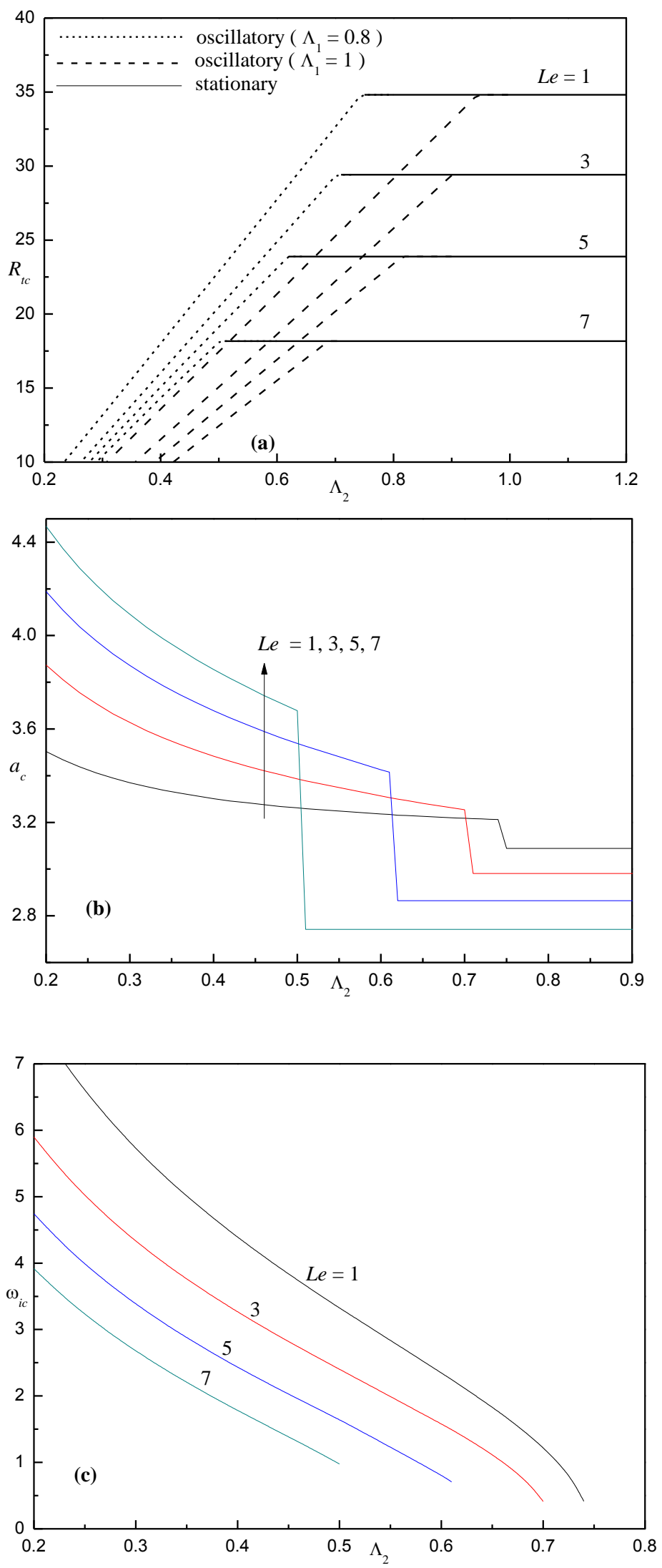

Fig. 8 Variation of (a) $R_{t c}$ (b) $a_{c}\left(\Lambda_{1}=0.8\right)$ (c) $\omega_{i c}\left(\Lambda_{1}=0.8\right)$ with $\Lambda_{2}$ for different values of Le when $N_{A}=1, R_{n}=2, M=1, N_{B}=0.01$. 